This is the accepted version of the article:

Martín-Illán J.Á., Rodríguez-San-Miguel D., Rodríguez-San-Miguel D., Franco C., Imaz I., Maspoch D., Maspoch D., Puigmartí-Luis J., Zamora F., Zamora F., Zamora F., Zamora F.. Green synthesis of imine-based covalent organic frameworks in water. Chemical Communications, (2020). 56. : 6704 - . 10.1039/d0cc02033h.

Available at: https://dx.doi.org/10.1039/d0cc02033h 


\section{Green Synthesis of Imine-based Covalent Organic Frameworks in Water}

Jesús Á. Martín-Illán, a David Rodríguez-San-Miguel,a,b Carlos Franco, ${ }^{\mathrm{b}}$ Inhar Imaz, ${ }^{\mathrm{c}}$ Daniel Maspoch, ${ }^{c, d}$ Josep Puigmartí-Luis, ${ }^{\text {b* }}$ and Félix Zamora ${ }^{a, e, f, g^{*}}$

Dynamic covalent bonds have been advantageously used to direct the synthesis of crystalline porous covalent organic frameworks (COFs). Unlike the standard synthetic protocols that involve harsh conditions, this work provides a high-yield "one-pot" green synthesis of imine-based COFs in water. Additionally, this aqueous synthesis can be performed under microwave conditions, considerably reducing the reaction time.

Covalent organic frameworks (COFs) are organic crystalline polymers showing permanent porosity. ${ }^{1,2}$ Modular chemistry concepts are behind the synthesis of these materials, producing programmed, extended and ordered structures, which properties can be fine-tuned based on the selection of molecular building blocks. ${ }^{3}$ Thus, the combination of the building blocks determines not only the COF architecture but also their physico-chemical properties. Indeed, COFs present interesting properties going from gas storage and separation ${ }^{4-6}$ to applications in optoelectronics, ${ }^{7}$ drug delivery, ${ }^{8,9}$ molecular separation, ${ }^{10,11}$ sensing, ${ }^{12,13}$ water decontamination ${ }^{14}$ and catalysis. ${ }^{15-17}$ A key factor to successfully control COF crystallinity is the use of reversible covalent bonds to connect the molecular building blocks while allowing error correction. ${ }^{2,18,19}$ Hence, the most typical reactions used to

a. Departamento de Química Inorgánica, Universidad Autónoma de Madrid, 28049 Madrid, Spain.Tel: 3491 4973962; E-mail: felix.zamora@uam.es.

b. Department of Chemistry and Applied Biosciences, Institute for Chemical and Bioengineering, ETH Zurich, Vladimir Prelog Weg 1, 8093 Zurich, Switzerland. c. Catalan Institute of Nanoscience and Nanotechnology (ICN2), CSIC and BIST Campus UAB, Bellaterra, 08193 Barcelona, Spain.

d. ICREA, Pg. Lluís Companys 23, 08010 Barcelona, Spain.

e. Instituto Madrileño de Estudios Avanzados en Nanociencia (IMDEA-Nanociencia), Cantoblanco, Madrid E-28049, Spain.

f. Institute for Advanced Research in Chemical Sciences (IAdChem). Universidad Autónoma de Madrid. 28049 Madrid, Spain.

g. Condensed Matter Physics Center (IFIMAC). Universidad Autónoma de Madrid. 28049 Madrid, Spain.

+ Electronic Supplementary Information (ESI) available: Additional experimental data and theoretical calculations. See DOI: 10.1039/x0xx00000x

produce COFs are based on condensation reactions between two different monomers. Initially, a large variety of boronbased COFs synthesized by the condensation reaction of two boronic acids or boronic acids with catechols were described. ${ }^{1,20}$
However, more recently, because of the enhanced chemical robustness, COFs produced by Schiff-base chemistry (e.g. iminebased COFs) have been largely developed. ${ }^{21}$

Currently, the most typical synthetic protocol to produce imine-based COFs requires using acid catalysts, solvothermal conditions (temperatures as high as $120-150^{\circ} \mathrm{C}$ ), long reaction times (48-72 hours) and high boiling point and hazardous organic solvents, such as mesitylene, 1,4-dioxane, $N, N$ dimethylformamide (DMF), $N, N$-dimethylacetamide (DMA), and $o$-dichlorobenzene (DCB), among others. ${ }^{21}$
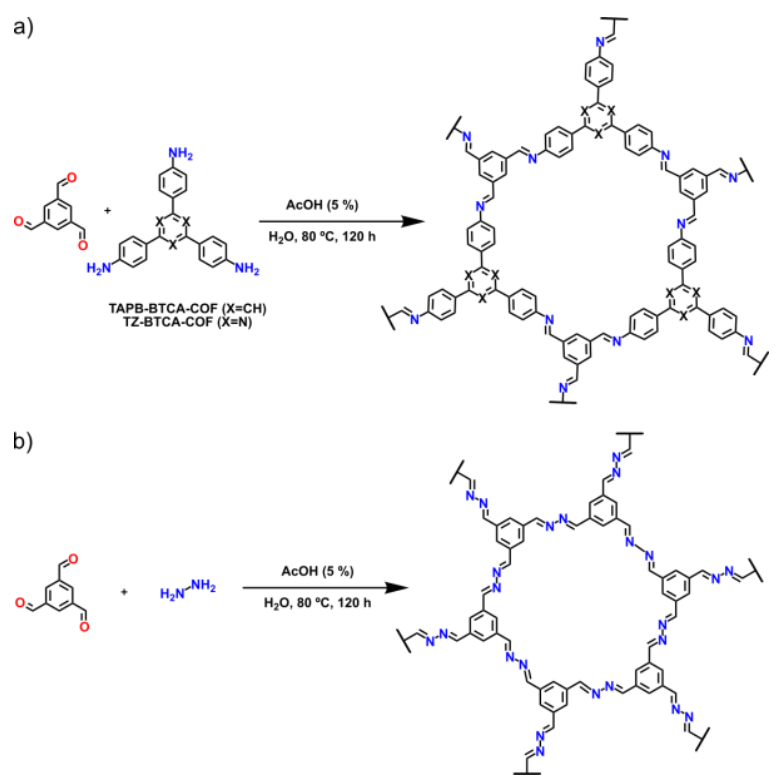

Scheme 1. Synthetic scheme of a) TAPB-BTCA-COF and TZ-BTCA-COF; and b) HZ-BTCACOF. 

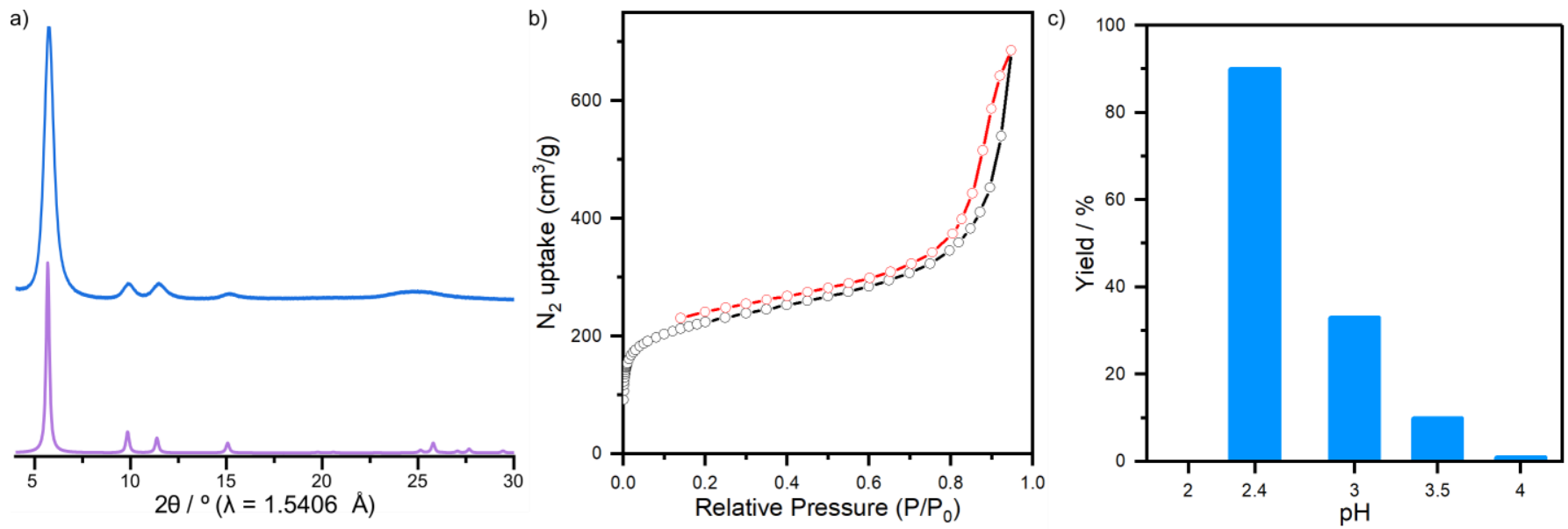

Fig. 1 a) Experimental (blue) and simulated (purple) PXRD patterns of TAPB-BTCA-COF. b) $\mathrm{N}_{2}$ adsorption-desorption isotherm of TAPB-BTCA-COF. Adsorption branch in black, desorption branch in red. c) Bar graph representing the yields of TAPB-BTCA-COF obtained at different pH values. At pH 2 no product was obtained.

In view of the great potential applications of these new porous materials, it will be highly desired to develop more sustainable procedures to prepare high-quality COFs, i.e. with a high porosity and crystallinity. Thus far, only a few reports have focused on green synthetic protocols for the production of COFs. ${ }^{22-25}$ For example, Banerjee et al. and Zhao et al. have recently reported the synthesis of keto-enamine-based COFs using a hydrothermal method. 26,27 However, these processes have been limited to this specific type of COFs. In fact, both authors explored the ability of their procedures to generate imine-linked COFs and concluded that the lack of hydroxyl groups in the aldehyde building block precluded the synthesis of COFs. This highlights the fact that the chemistry of imine and keto-enamine-linked COFs is significantly different, and that new synthetic protocols are needed to synthesize imine-linked COFs in more environmentally friendly conditions.

Herein we report a simple protocol for preparing iminebased COFs using water as solvent and a limited amount of acetic acid as catalyst at relatively low temperatures. The soformed COFs are obtained in high yield showing good crystallinity and porosity. Additionally, we also demonstrate that the same protocol can be carried out under microwave irradiation, significantly reducing the reaction time from some days down to hours.

We started with the aqueous synthesis of the imine-based TAPB-BTCA-COF made of 1,3,5-benzenetricarbaldehyde (BTCA) and 1,3,5-tris-(4-aminophenyl)benzene (TAPB) (Scheme 1a). TAPB-BTCA-COF was synthesized by mixing two aqueous solutions, one containing BTCA and the other containing TAPB and acetic acid. The resulting mixture $(\mathrm{pH} 2.4)$ was then heated at $80{ }^{\circ} \mathrm{C}$ for 5 days (ESI for experimental details). Fourier transform infrared spectroscopy (FT-IR), ${ }^{13} \mathrm{C} \mathrm{CP}-\mathrm{MAS}$ solid-state NMR and powder X-ray diffraction (PXRD) of the resulting solid corroborated the formation of TAPB-BTCA-COF. FT-IR spectrum showed the presence of imine bonds through the appearance of the characteristic $\mathrm{C}=\mathrm{N}$ stretching at $1624 \mathrm{~cm}^{-1}$ (Fig. S1, ESI + ). Moreover, the characteristic vibrations of the amino and carbonyl groups of the precursors were not appreciated, indicating the completion of the reaction. ${ }^{13} \mathrm{C}$ CP-MAS solidstate NMR spectrum also confirmed the formation of imine bonds showing the characteristic signal at $155 \mathrm{ppm}$ that corresponds to the imine carbon (Table $\mathrm{S} 1$ and Fig. S2, ESI + ). Fig. 1a shows the PXRD pattern of TAPB-BTCA-COF, which matches with the simulated pattern in AA eclipsed stacking model. ${ }^{28}$ The intense first peak was attributed to the strongly reflecting (100) plane. The thermogravimetric analysis (TGA) of TAPB-BTCA-COF in $\mathrm{N}_{2}$ atmosphere demonstrated its thermal stability up to 500 ${ }^{\circ} \mathrm{C}$ (Fig. S3, ESI + ). Note here that all these data are in good agreement with previously reported values for TAPB-BTCA-COF synthesized under standard solvothermal conditions and using hazardous organic solvents. ${ }^{29}$ Additionally, the microporosity of TAPB-BTCA-COF was confirmed by $\mathrm{N}_{2}$ adsorption analysis at 77 $\mathrm{K}$ (Fig. $1 \mathrm{~b}$ and Table S7, ESI + ), from which a BET surface area $\left(\mathrm{SA}_{\mathrm{BET}}\right.$ ) of $806 \mathrm{~m}^{2} \mathrm{~g}^{-1}$ was calculated (Fig. S4, ESI $)$ ). To the best of our knowledge, this $\mathrm{SA}_{\mathrm{BET}}$ value of TAPB-BTCA-COF is one of the highest reported, even when compared to TAPB-BTCA-COF synthesized using tetrahydrofuran:mesitylene mixtures in solvothermal conditions. ${ }^{30}$ The collected isotherm is a type IV isotherm according to IUPAC classification, presenting a hysteresis loop. This behaviour can be attributed to the presence of some mesoporosity resulting from the nanocrystal assembly (Fig. S5, ESIt) and/or to some structural swelling; as sometimes observed in this type of materials. ${ }^{31,32}$

In order to further understand the factors that affect the generation of imine-based COFs under these mild conditions, we evaluated the influence of $\mathrm{pH}$ in the synthesis of TAPBBTCA-COF. To this end, we reproduced its synthesis in water at several $\mathrm{pH}$ values from 2 to 4 by adjusting the amount of acetic acid (ESI for experimental details). Fig. 1c indicates the clear dependence that the imine-condensation reaction has with $\mathrm{pH}$, where a maximum yield was obtained at $\mathrm{pH}$ value 2.4 (see also Table S3). Even though the reaction yield decreased by $30 \%$, we also observed that the TAPB-BTCA-COF powder obtained at $\mathrm{pH}$ 3 showed a good value of $S A_{B E T}$ above $750 \mathrm{~m}^{2} \mathrm{~g}^{-1}$ (Fig. $\mathrm{S} 6$ and Table S7, ESIt). Surprisingly, the $\mathrm{SA}_{\mathrm{BET}}$ was dramatically decreased at $\mathrm{pH} 3.5$, where values around $150 \mathrm{~m}^{2} \mathrm{~g}^{-1}$ were 
found. Remarkably, in all the conditions screened, the synthesized COF powders showed clear TAPB-BTCA-COF diffraction peaks (Fig. S7, ESI†).

We attribute the changes of the reaction yield at different $\mathrm{pHs}$ to the degree of protonation of TAPB in the aqueous medium. While BTCA was soluble in the complete $\mathrm{pH}$ range studied, the solubility of TAPB was $\mathrm{pH}$ dependent (Table S5). Note that TAPB is a weak triprotic base with a large aromatic skeleton. Accordingly, even though the neutral amine has an extremely low solubility in water, its charged ammonium cations are the predominant species in highly acidic solutions and are readily dissolved in water. Thus, at $\mathrm{pH} \mathrm{4,} \mathrm{a} \mathrm{TAPB}$ concentration of only $31 \mu \mathrm{M}$ was available to react with BTCA (Table S5), and therefore, the formation of TAPB-BTCA-COF was almost precluded at this $\mathrm{pH}$. At $\mathrm{pH}<3.5$, the solubility of TAPB increased gradually, also increasing the yield of the reaction until complete solubilisation was achieved at an optimal $\mathrm{pH}$ of 2.4 (Table S3), with a maximum yield of $97 \%$. However, lowering the $\mathrm{pH}$ beyond this point resulted in a complete inhibition of the reaction, with no solid precipitating from the reaction mixture, which remained clear and colourless after 5 days at $80^{\circ} \mathrm{C}$. This is in agreement with the work of Banerjee et $a l,{ }^{26}$ who observed that it is not possible to obtain imine-based COFs in aqueous solutions with a high concentration of acetic acid even using hydrothermal conditions. The lack of reaction at this low $\mathrm{pH}$ value can be easily rationalized considering again the basic nature of TAPB and the mechanism of the imine formation. We found out that the $\mathrm{pK}_{\mathrm{a}}$ value for the first acidbase equilibrium of the TAPB system (that is, from the fully protonated triammonium ion to the doubly protonated molecule) is close to 3.5 (Fig. S8). This implies that, at $\mathrm{pH} \mathrm{2,} \mathrm{only}$ $3 \%$ of the TAPB molecules will have one non-protonated amine that can act as a nucleophile. This is of great importance, since the crucial step for the formation of an imine bond from an aldehyde and an amine is the nucleophilic attack of the nitrogen of the amino group to the carbon of the carbonyl group. Therefore, at $\mathrm{pH} 2$, even though TAPB is completely dissolved in the reaction mixture, it cannot react with BTCA because its reactivity is greatly decreased due to the protonation of the amino groups. Hence, these results show that, in order to synthesise imine-based COFs in water, it is necessary to find the $\mathrm{pH}$ range in which both amine and aldehyde building blocks are completely dissolved but also reactively active to trigger the condensation reaction. On the other hand, the change of surface area is likely related to the presence of undissolved monomers, as also suggested by Zhao et al. for keto-enamine COFs. ${ }^{27}$ In order to further support this hypothesis, we prepared TAPB-BTCA-COF at $\mathrm{pH} 2.4$ doubling the concentration of monomers (ESI for experimental details). Under these conditions, the yellow solid obtained showed a large decrease in crystallinity (Fig. S10) and a loss of porosity (Fig. S11).

Having successfully produced TAPB-BTCA-COF in water, we extended our aqueous method to the synthesis of HZ-BTCA-COF and TZ-BTCA-COF, where $\mathbf{H Z}$ and TZ stand for hydrazine and 2,4,6-tris(4-aminophenyl)-1,3,5-triazine, respectively (Scheme
1). To this end, we employed similar conditions to the ones described above where the $\mathrm{pH}$ was adjusted to obtain the maximum yield in both COFs synthesis (ESI for experimental details). Note here that a small amount of DMSO (0.3\% in vol.) was used to facilitate the solubilization of $\mathrm{TZ}$ in water during the synthesis of TZ-BTCA-COF (for further details see ESI). ${ }^{33}$ Remarkably, FT-IR spectra (Figs S12 and S13, ESI+), ${ }^{13}$ C CP-MAS solid-state NMR spectra (Tables S5 and S6 and Figs S14 and S15, ESI') and TGA (Figs S16 and S17, ESI + ) of HZ-BTCA-COF and TZBTCA-COF matched with the previously reported data for both COFs synthesized under conventional solvothermal conditions. 5,34 Moreover, the PXRD patterns of both synthesized COFs matched with the corresponding simulated patterns in AA eclipsed stacking models (Fig. 2a).5,34 In fact, the SABET values measured were $1366 \mathrm{~m}^{2} \mathrm{~g}^{-1}$ for HZ-BTCA-COF (Figs. S18 and S19) and $1533 \mathrm{~m}^{2} \mathrm{~g}^{-1}$ for TZ-BTCA COF (Figs. S20 and S21). These values are comparable to those previously reported for both COFs obtained by solvothermal synthesis in mesitylene:dioxane mixtures. 5,34
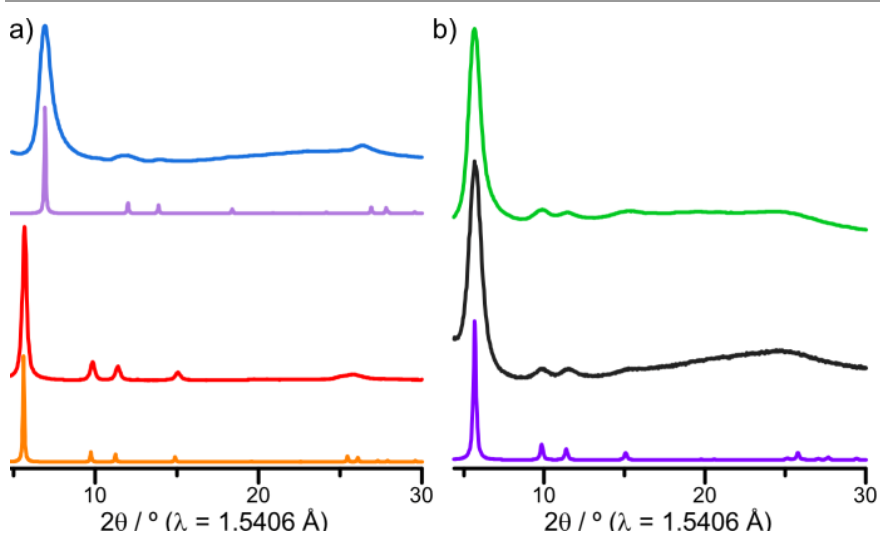

Fig. 2 a) PXRD patterns of HZ-BTCA-COF obtained in water (blue) and simulated (purple). and of TZ-BTCA-COF obtained in water (red) and simulated (orange). b) PXRD patterns of TAPB-BTCA-COF obtained in water close to ambient conditions (RT TAPB-BTCA-COF, green), under hydrothermal MW conditions (MW TAPB-BTCA-COF; black) and simulated (purple).

Finally, to further reduce the energy costs and shorten the reaction times, we prepared TAPB-BTCA-COF in water close to room temperature conditions (RT TAPB-BTCA-COF) and under hydrothermal microwave radiation (MW TAPB-BTCA-COF). For the first case, RT TAPB-BTCA-COF could be prepared (90\% yield) by heating an aqueous solution $(\mathrm{pH} 2.4)$ of TAPB and BTCA in the presence of acetic acid and at $35^{\circ} \mathrm{C}$ for 5 days. Interestingly, this COF showed a crystallinity (Fig. 2b) and SA $\mathrm{AET}_{\mathrm{BET}}$ of $803 \mathrm{~m}^{2} \mathrm{~g}^{-1}$ (Table S7 and Figs. S23 and S24, ESIT) similar to the same material synthesized at $80^{\circ} \mathrm{C}$.

Also, microwave radiation was proven useful for the aqueous synthesis of imine-based COFs. Here, we heated an aqueous mixture $(\mathrm{pH} 2.4)$ containing both precursors and acetic acid at $80^{\circ} \mathrm{C}$ in a $200 \mathrm{~W}$ microwave oven (ESI for experimental details). MW TAPB-BTCA-COF was obtained in 5 hours with an equivalent yield $(85 \%)$ to the reaction carried out for 5 days using conventional heating. The FT-IR spectrum (Fig. S25) and PXRD pattern (Fig. 2b) of MW TAPB-BTCA-COF were similar to 
those previously obtained for TAPB-BTCA-COF. Moreover, MW TAPB-BTCA-COF showed a SA BET $_{\text {of }} 566 \mathrm{~m}^{2} \mathrm{~g}^{-1}$ (Table $\mathrm{S7}$ and Figs. S26 and S27, ESIt). This value is slightly smaller than the previous TAPB-BTCA-COF synthesized in water, but it is in the range of that synthesized in organic solvents, ${ }^{28,35}$ including mesitylene:dioxane mixtures. ${ }^{29,30}$

This work provides a simple one-step green method to synthesize imine-based COFs in water with good quality in terms of crystallinity and porosity; in high yields; and avoiding the use of harmful organic solvents and high temperatures. The versatility of this method has been corroborated preparing three structurally and chemically different imine-based COFs. We also showed that imine-based COFs can also be produced in water decreasing the reaction temperature down to $35^{\circ} \mathrm{C}$, or by using microwave heating with a significant reduction of the reaction time, from several days down to 5 hours. The results obtained pave the way for large-scale synthesis of imine-based COFs in water presenting a more sustainable method that avoids the use of organic solvents, otherwise required for COF synthesis.

\section{Conflicts of interest}

There are no conflicts to declare.

\section{Acknowledgements}

This work was supported by the EU (ERC-2015-STG microCrysFact 677020 and ERC-Co 615954), Swiss National Science Foundation (Project no. 200021_160174 and 200021_181988), ETH Zürich, Catalan AGAUR (project 2017 SGR 238), and MICINN (MAT2016-77608-C3-1P and RTI2018095622-B-I00). It was also funded by the CERCA Program/Generalitat de Catalunya. ICN2 is supported by Severo Ochoa program from MINECO (SEV-2017-0706).

\section{References}

1 A. P. Côté, A. I. Benin, N. W. Ockwig, M. O'Keeffe, A. J. Matzger and O. M. Yaghi, Science, 2005, 310, 1166-1170.

2 X. Feng, X. Ding and D. Jiang, Chem. Soc. Rev., 2012, 41, 6010.

3 S.-Y. Ding and W. Wang, Chem Soc Rev, 2013, 42, 548-568.

4 S. Yuan, X. Li, J. Zhu, G. Zhang, P. Van Puyvelde and B. Van der Bruggen, Chem. Soc. Rev., 2019, 48, 2665-2681.

5 Z. Li, X. Feng, Y. Zou, Y. Zhang, H. Xia, X. Liu and Y. Mu, Chem Commun, 2014, 50, 13825-13828.

6 C. J. Doonan, D. J. Tranchemontagne, T. G. Glover, J. R. Hunt and O. M. Yaghi, Nat. Chem., 2010, 2, 235-238.

7 M. Calik, F. Auras, L. M. Salonen, K. Bader, I. Grill, M. Handloser, D. D. Medina, M. Dogru, F. Löbermann, D. Trauner, A. Hartschuh and T. Bein, J. Am. Chem. Soc., 2014, 136, 17802-17807.

8 L. Bai, S. Z. F. Phua, W. Q. Lim, A. Jana, Z. Luo, H. P. Tham, L. Zhao, Q. Gao and Y. Zhao, Chem. Commun., 2016, 52, 41284131.
9 Q. Fang, J. Wang, S. Gu, R. B. Kaspar, Z. Zhuang, J. Zheng, H. Guo, S. Qiu and Y. Yan, J. Am. Chem. Soc., 2015, 137, 83528355.

10 J. Shen, R. Zhang, Y. Su, B. Shi, X. You, W. Guo, Y. Ma, J. Yuan, F. Wang and Z. Jiang, J. Mater. Chem. A, 2019, 7, 1806318071.

11 H. Yang, L. Yang, H. Wang, Z. Xu, Y. Zhao, Y. Luo, N. Nasir, Y. Song, H. Wu, F. Pan and Z. Jiang, Nat. Commun., 2019, 10, 2101.

12 P. Albacete, A. López-Moreno, S. Mena-Hernando, A. E. Platero-Prats, E. M. Pérez and F. Zamora, Chem. Commun., 2019, 55, 1382-1385.

13 L. Ascherl, E. W. Evans, M. Hennemann, D. Di Nuzzo, A. G. Hufnagel, M. Beetz, R. H. Friend, T. Clark, T. Bein and F. Auras, Nat. Commun., 2018, 9, 3802.

14 L. Merí-Bofí, S. Royuela, F. Zamora, M. L. Ruiz-González, J. L. Segura, R. Muñoz-Olivas and M. J. Mancheño, J. Mater. Chem. A, 2017, 5, 17973-17981.

15 Y. Han, M. Zhang, Y.-Q. Zhang and Z.-H. Zhang, Green Chem., 2018, 20, 4891-4900.

16 P.-F. Wei, M.-Z. Qi, Z.-P. Wang, S.-Y. Ding, W. Yu, Q. Liu, L.-K Wang, H.-Z. Wang, W.-K. An and W. Wang, J. Am. Chem. Soc. 2018, 140, 4623-4631.

17 S. M. J. Rogge, A. Bavykina, J. Hajek, H. Garcia, A. I. OlivosSuarez, A. Sepúlveda-Escribano, A. Vimont, G. Clet, P. Bazin, F. Kapteijn, M. Daturi, E. V. Ramos-Fernandez, F. X. Llabrés i Xamena, V. Van Speybroeck and J. Gascon, Chem. Soc. Rev., 2017, 46, 3134-3184.

18 Y. Jin, Y. Hu and W. Zhang, Nat. Rev. Chem., 2017, 1, 0056.

19 C. S. Diercks and O. M. Yaghi, Science, 2017, 355, eaal1585.

20 E. L. Spitler and W. R. Dichtel, Nat. Chem., 2010, 2, 672-677.

21 J. L. Segura, M. J. Mancheño and F. Zamora, Chem. Soc. Rev., 2016, 45, 5635-5671.

22 X. Li, Y. Qi, G. Yue, Q. Wu, Y. Li, M. Zhang, X. Guo, X. Li, L. Ma and S. Li, Green Chem., 2019, 21, 649-657.

23 F. Zhang, J. Zhang, B. Zhang, X. Tan, D. Shao, J. Shi, D. Tan, L. Liu, J. Feng, B. Han, G. Yang, L. Zheng and J. Zhang, ChemSusChem, 2018, 11, 3576-3580.

24 S. Karak, S. Kandambeth, B. P. Biswal, H. S. Sasmal, S. Kumar, P. Pachfule and R. Banerjee, J. Am. Chem. Soc., 2017, 139, 1856-1862.

25 W. Dai, F. Shao, J. Szczerbiński, R. McCaffrey, R. Zenobi, Y. Jin, A. D. Schlüter and W. Zhang, Angew. Chem. Int. Ed., 2016, 55, 213-217.

26 J. Thote, H. Barike Aiyappa, R. Rahul Kumar, S. Kandambeth, B. P. Biswal, D. Balaji Shinde, N. Chaki Roy and R. Banerjee, IUCrJ, 2016, 3, 402-407.

27 J. Lu, F. Lin, Q. Wen, Q.-Y. Qi, J.-Q. Xu and X. Zhao, New J. Chem., 2019, 43, 6116-6120.

28 A. de la Peña Ruigómez, D. Rodríguez-San-Miguel, K. C. Stylianou, M. Cavallini, D. Gentili, F. Liscio, S. Milita, O. M. Roscioni, M. L. Ruiz-González, C. Carbonell, D. Maspoch, R. Mas-Ballesté, J. L. Segura and F. Zamora, Chem. - Eur. J., 2015, 21, 10666-10670.

29 D. Rodríguez-San-Miguel, A. Yazdi, V. Guillerm, J. PérezCarvajal, V. Puntes, D. Maspoch and F. Zamora, Chem. Eur. J., 2017, 23, 8623-8627.

30 M. C. Daugherty, E. Vitaku, R. L. Li, A. M. Evans, A. D. Chavez and W. R. Dichtel, Chem. Commun., 2019, 55, 2680-2683.

31 V. Guillerm, Ł. J. Weseliński, M. Alkordi, M. I. H. Mohideen, Y. Belmabkhout, A. J. Cairns and M. Eddaoudi, Chem. Commun., 2014, 50, 1937.

32 R. Dawson, A. I. Cooper and D. J. Adams, Prog. Polym. Sci., 2012, 37, 530-563.

33 V. S. Vyas, F. Haase, L. Stegbauer, G. Savasci, F. Podjaski, C. Ochsenfeld and B. V. Lotsch, Nat. Commun., 2015, 6, 8508. 
34 Q. Gao, L. Bai, X. Zhang, P. Wang, P. Li, Y. Zeng, R. Zou and Y. Zhao, Chin. J. Chem., 2015, 33, 90-94.

35 D. Rodríguez-San-Miguel, A. Abrishamkar, J. A. R. Navarro, R. Rodriguez-Trujillo, D. B. Amabilino, R. Mas-Ballesté, F.

Zamora and J. Puigmartí-Luis, Chem. Commun., 2016, 52, 9212-9215. 